\title{
International Randomised Controlled Trial for the Treatment of Newly Diagnosed Ewing Sarcoma Family of Tumours - EURO EWING 2012
}

Jennifer Anne Birmingham

University of Birmingham

Veronica Moroz

University of Birmingham

Perrine Marec-Berard

Centre Leon Berard

Nathalie Gaspar

Institut Gustave Roussy

Valerie Laurence

Institut Curie

Javier Martin-Broto

GEIS

Ana Sastre

GEIS

Hans Gelderblom

EORTC

Cormac Owens

$\mathrm{OLCH}$

Sophie kaiser

CLB

Melissa Fernández-Pinto

GEIS

Nicola Fenwick

CRCTU

Abigail Evans

UCL

Sandra Strauss

UCL

Jeremy Whelan

UCLH

Keith Wheatley 
UoB

Bernadette Brennan ( $\sim$ Bernadette.Brennan@mft.nhs.uk)

\section{Study protocol}

Keywords: Ewing Sarcoma family of tumours, randomised controlled trial

Posted Date: July 22nd, 2019

DOl: https://doi.org/10.21203/rs.2.11789/v1

License: (c) (1) This work is licensed under a Creative Commons Attribution 4.0 International License. Read Full License

Version of Record: A version of this preprint was published on January 17th, 2020. See the published version at https://doi.org/10.1186/s13063-019-4026-8. 


\section{Abstract}

Abstract Background Despite multiple randomised trials in newly diagnosed Ewing sarcoma family of tumours (ESFT), over many years, and involving many international co-operative groups, the outcomes for all stages of disease have plateaued. Internationally, the standard treatment of ESFT is not defined, and there is a need to add new agents other than conventional chemotherapy to improve outcomes. This trial will compare two different induction/consolidation chemotherapy regimens vincristine, ifosfamide, doxorubicin and etoposide (VIDE) induction and vincristine, actinomycin D, ifosfamide or cyclophosphamide, or Busulfan and Mephalan (VAI/VAC/BuMel) consolidation compared with vincristine, doxorubicin, cyclophosphamide, ifosfamide and etoposide (VDC/IE) induction and ifosfamide and etoposide, vincristine and cyclophosphamide, vincristine, actinomycin D and ifosfamide, or Busulfan and Mephalan (IE/VC/VAl/ BuMel) consolidation- randomisation 1 (R1). A second randomisation (R2) will determine whether the addition of zoledronic acid to consolidation chemotherapy, as assigned at R1, is associated with improved clinical outcome. Methods EURO EWING 2012 is an international, multicentre, phase III, open-label randomised controlled trial. There are two randomisations: R1 and R2. Patients are randomised at two different time points, at entry to the trial (R1) and following local control therapy (R2). The primary outcome measure is event-free survival. The secondary outcome measures include overall survival, adverse events and toxicity, histological response of the primary tumour, response of the primary tumour, regional lymph nodes and/or metastases, and achievement of local control at the end of treatment. Discussion This study will establish which is the "standard regimen" of chemotherapy, taking into account both clinical outcomes and toxicity. This will form the chemotherapy backbone for future interventional studies where we may want to add new targeted agents. It will also determine the role of zoledronic acid, in conjunction with the separate EE2008 trial. Any trial in ESFT needs to take into account the rarity of the tumour and that international co-operation is needed to provide answers in a timely manner. Trial registration Registered with EudraCT number 2012-002107-17 on 26th February 2012. Registered with ISRCTN number 54540667 on 4th November 2013.

\section{Background And Rationale}

The Ewing sarcoma family of tumours (ESFT) usually arise in skeletal sites in children and young people and consist of small, round malignant cells that may exhibit varying degrees of neural differentiation. ESFT are characterised by a re-arrangement involving chromosome 22, with an 11;22 translocation detectable in more than $95 \%$ of cases [1-7]. The gene rearrangement results in the production of a transcription factor - in the majority, EWS-FLI1 transcription.

Most ESFT arise in bony sites. Staging procedures identify approximately $30 \%$ of patients with detectable metastases at diagnosis. Since chemotherapy was introduced routinely in the 1970s, cure rates have dramatically improved and with current multimodal programmes including combination chemotherapy, surgery and radiotherapy the 5-year survival rate for localised ESFT is about $65 \%$ with chemotherapy regimens including actinomycin $\mathrm{D}$, doxorubicin, etoposide, cyclophosphamide, vincristine and ifosfamide, with different doses and schedules of administration ESFT with lung-only metastases treated with 
conventional chemotherapy experience a 3 year event-free survival (EFS) of approximately $30 \%$ while for those patients with disseminated disease, the prognosis remains very poor, the EURO-E.W.I.N.G. 99 trial demonstrating overall survival at 3 years of $29 \%$ [8].

Internationally, a single standard chemotherapy treatment for ESFT is not defined. The EURO-E.W.I.N.G. 99 trial employed VIDE induction chemotherapy (6 cycles of vincristine, ifosfamide, doxorubicin and etoposide given approximately every 3 weeks prior to local control), followed by risk adapted randomised treatment of either vincristine, actinomycin D and ifosfamide or cyclophosphamide (VAI/VAC) as consolidation chemotherapy or high-dose busulfan/melphalan. The toxicity of VIDE induction chemotherapy has been published [9]. In summary, $12 \%$ had grade III or IV stomatitis, $3 \%$ had cardiac left ventricular dysfunction as determined by fractional shortening, there were 5 toxic related deaths out of 851 patients giving a rate of $0.6 \%$, and grade II, III and IV infections occurred in $40 \%, 9 \%$ and $0.6 \%$ respectively. As yet, the data on second malignant neoplasms (SMNs) have not been published but, in the EURO-E.W.I.N.G. 99 trial between 1 September 2001 and 1 September 2005, there have been 5 SMNs (2 leukaemias and 3 solid tumours) in the 462 registered patients with localised disease (Marie-Cécile Le Deley, personal communication).

The other widely used treatment regimen for ESFT, employed mainly in the USA, is that from the Children's Oncology Group (COG) AEWS0031 trial [10]. In this study, patients with localised ESFT received alternating cycles of vincristine-doxorubicin-cyclophosphamide and ifosfamide-etoposide (VDC/IE) as induction chemotherapy, and alternating cycles of ifosfamide-etoposide and vincristinecyclophosphamide (IE/VC) as consolidation chemotherapy. There was an upfront randomisation to compare 3-weekly cycles of this treatment (standard arm) with 2-weekly cycles (experimental arm). There was significantly superior EFS of $73 \%$ in the compressed 2-weekly VDC/IE/VC, compared to $65 \%$ in the standard arm $(p=0.048)$, and also improved overall survival, $83 \%$ and $77 \%$ respectively $(p=0.056)$. This compressed induction regimen has now become the standard regimen for localised ESFT in the USA. Regarding short term toxicity, there was one toxic death in the compressed arm B. In arm B, despite compression of the chemotherapy cycles, stomatitis occurred in $3 \%$ and colitis or typhlitis in $0.4 \%$ of chemotherapy cycles. There were no episodes of cardiac left ventricular dysfunction and grade III/IV infectious toxicities occurred as follows: febrile neutropenia $7 \%$, infection with grade $3 / 4$ neutropenia $5 \%$, infection without neutropenia $2 \%$ and infection (white cell count unknown) $0.3 \%$. Therefore a randomisation at diagnosis between VIDE and VAI/VAC versus VDC/IE/VC is necessary to establish which is the regimen of choice, taking account of both clinical outcome (EFS and OS) and toxicity.

Bisphosphonates, a group of compounds which inhibit bone resorption, have been used for the treatment of bone metastases in patients with breast cancer, multiple myeloma and prostate cancer [11-13]. In vitro and in vivo data have also proven the anti-tumour activity of Nitrogen-containing bisphosphonates ( $\mathrm{N}$ BPs) against ESFT cells. The N-BPs pamidronate inhibits growth in eight different ESFT cell lines via inhibition of the mevalonate pathway [14]. Zhou et al showed significant inhibition in the development of bone metastases after injection of the bisphosphonate zoledronic acid in vivo; N-BPs induced apoptosis and inhibited osseous metastases [15]. Zoledronic acid has a direct inhibitory effect on the growth of 
ESFT cells in vitro which is induced by apoptosis associated with caspase 3 activation and cell cycle arrest in S phase. This effect was enhanced by alkylating agents. Using an in vivo mouse model, zoledronic acid exerted a strong inhibitory effect on the growth of bone ESFT and little effect on the growth of intramuscularly injected ESFT. When combined with ifosfamide, zoledronic acid exerted synergistic effects in the soft tissue model: its combination with one cycle of ifosfamide resulted in an inhibitory effect similar to three cycles of ifosfamide alone [16].

Although there are no clinical studies of zoledronic acid in ESFT, except for a single case report of a multiple relapsed patient responding to zoledronic acid with third line chemotherapy, its low toxicity profile with conventional chemotherapy and the growing body of evidence for the use of bisphosphonates for the treatment of bone metastases in other cancers described above provide ample justification to examine the value of zoledronic acid in a clinical trial.

Despite ESFT being the second commonest malignant bone tumour in children, adolescents and young adults, they remain rare tumours (less than 70 cases per year in the UK) and hence any randomised trials must be international. The EURO EWING Consortium (EEC) is a partnership of specialists in 15 European countries working together to improve the outcome ESFT. The activities of the EEC are funded by the European Union for five years and include two clinical trials, translational research and the strengthening of both patient and public involvement and the ethical process. This article presents the study protocol of the EEC clinical trial; International Randomised Controlled Trial for the Treatment of Newly Diagnosed Ewing Sarcoma Family of Tumours (EURO EWING 2012).

\section{Methods}

\section{Design and Objectives}

EURO EWING 2012 (EE2012) is an international, multicentre, phase III, open-label randomised controlled trial. There are two randomisations: patients are randomised, at entry (Randomisation $1 ; \mathrm{R} 1$ ) and then following local control therapy (Randomisation 2; R2). The trial schema is shown in figure 1.

The objective of the induction/consolidation chemotherapy R1 is to compare:

VIDE as induction chemotherapy and VAI/VAC /BuMel as consolidation chemotherapy (Arm A) with VDC/IE induction and IE/VC or VAI/BuMel consolidation chemotherapy (Arm B) as first line treatment in all patients with ESFT, with respect to clinical outcome and toxicity.

The objective of the zoledronic acid randomisation (R2) is to determine whether the addition of zoledronic acid to the consolidation chemotherapy assigned at R1 is associated with improved clinical outcome in patients in the EE2012 trial.

The third objective is, through the biological studies embedded in EE2012, to identify informative prognostic biomarkers for assessment of disease status and response at diagnosis and throughout the 
disease course. Whether they are predictive of response to therapy and may be used to improve stratification of patients and whether they might predict those patients that may not tolerate a particular therapy will also be explored.

\section{Figure 1: Trial Schema}

\section{Outcome measures}

The primary outcome measure is EFS. EFS is defined as the time from randomisation to first event, where an event is progression without complete remission, recurrence (following complete or partial remission), second malignancy or death. Patients who do not have an event by the end of the follow up period will be censored at their last follow-up date, and patients lost to follow-up without an event will be censored at the date of their last consultation.

The secondary outcome measures are:

- Overall Survival (OS) is defined as the time from randomisation to death, irrespective of the cause. Surviving patients will be censored at their last follow-up date,

- Adverse events and toxicity, defined by $\mathrm{NCl}$ Common Terminology Criteria for Adverse Events (CTCAE) v4.0,

- Histological response of the primary tumour to induction chemotherapy if surgery is performed as local control defined as the percentage of viable tumour cells in the resected primary tumour specimen,

- Response of the primary tumour, regional lymph nodes and/or metastases using the change in the volume of the primary tumour, the number of lung and/or pleural and other metastases, and /or the diameter of the largest node (or group if not separate),

- Achievement of local control at the end of treatment as defined by either complete surgical resection following induction chemotherapy, no measurable disease as assessed by end of treatment MRI or CT scan or no increase in measurable residual tumour over a six month period from the end of treatment,

- Growth parameters and jaw/ear osteonecrosis (R2 only) will be assessed using patient's height measured at baseline, treatment and throughout follow up for all patients who enter the second 
randomisation and who are less than 18 years of age at entry. Whether jaw and ear osteonecrosis occurred will be recorded at the end of or during treatment for all patients who were randomised to R2.

\section{Recruitment and randomisation}

All eligible patients with ESFT at participating centres are invited to take part in the trial. EEC partner organisations act as national coordinating centres (NCC) and identify participating centres within their country/countries. The University of Birmingham is the Coordinating Sponsor and also undertakes the responsibilities of NCC in the UK. One hundred and ten participating centres are taking part across 10 countries (Belgium, Czech Republic, Denmark, France, Hungary, Ireland, Netherlands, Spain, Switzerland and United Kingdom). Patients enter the trial via R1 and, if following induction chemotherapy they fulfil the R2 eligibility criteria, are then asked to participate in R2.

Patients are eligible if all of the trial inclusion criteria are met and none of the exclusion criteria apply. The eligibility criteria originally excluded patients with extrapulmonary metastatic disease, but this was amended in September 2016 (the exact date of implementation in each country varies as it was dependent upon gaining country specific regulatory approvals). The current eligibility criteria for R1 and R2 are shown in table 1.

\section{Table 1: Inclusion and exclusion criteria}

For each randomisation, patients are allocated in a 1:1 ratio to the two arms. Randomisation is performed by staff at participating centres online using randomisation function of the electronic remote data capture system (eRDC) designed and maintained by the Coordinating Sponsor.

The $\mathrm{R} 1$ randomisation is stratified by age at randomisation ( $<14$ years; $\geq 14$ years), sex, disease type (absence of metastases or involvement of lymph nodes only; lung or pleural metastases only; other metastases), volume of tumour at diagnosis $(<200 \mathrm{ml} ; \geq 200 \mathrm{ml})$ and country (UK, France or other) to ensure that there is a balance between treatments within the strata defined by these key prognostic factors.

The $\mathrm{R} 2$ randomisation is stratified by allocated treatment in $\mathrm{R} 1$, age at $\mathrm{R} 1$ randomisation ( $<14$ years; $\geq 14$ years), sex, disease status (localised disease or regional lymph node involvement of lymph nodes only at diagnosis and good risk after induction, localised disease or regional lymph node involvement only at diagnosis and of lymph nodes only poor risk after induction, lung or pleural metastases at diagnosis, other metastasis at diagnosis), and country (UK, France or other).

\section{Trial Treatment.}

Randomisation R1; At trial entry patients are randomised to one of the following treatment arms:

\section{- Arm A (VIDE strategy): VIDE induction; VAI/VAC/BuMel consolidation}

Induction chemotherapy:
6 cycles of VIDE

Page $7 / 16$ 
Consolidation chemotherapy: $\quad 1$ cycle of VAI plus 7 cycles of VAC

(good risk localised disease)- R2 VAC

OR

1 cycle VAl plus one cycle of BuMel (poor risk localised disease without contraindication to BuMel)

OR

8 cycles of VAI (poor risk localised disease with contraindication to BuMel, and/or regional lymph node(s) involvement and/or metastatic disease) - R2 VAI

OR

- Arm B (VDC/IE strategy): VDC/IE induction; IE/VC/VAI/BuMel consolidation

Induction chemotherapy: $\quad 9$ cycles of alternating VDC and IE

Consolidation chemotherapy: 5 cycles of alternating IE and VC

- R2 IE/VC (good risk localised disease, and/or regional lymph node(s) involvement and/or metastatic disease, or poor risk localised disease with contraindication to BuMel

OR

1 cycle VAl plus BuMel (poor risk localised disease without contraindication to BuMel)

Randomisation R2; Following induction chemotherapy, patients who fulfil the eligibility criteria for R2 and consent to take part in the randomisation will receive consolidation chemotherapy as allocated at trial entry and be randomised to receive either:

- 9 cycles of zoledronic acid following the first cycle of consolidation chemotherapy

OR

- No zoledronic acid

A summary of the enrolment, interventions and the main assessments is shown in figure 2, and a SPIRIT checklist is supplied as supplementary file 1 . The full schedule of treatments is provided in supplementary table 1 . 
Figure 2: Summary schedule of enrolment, interventions, and assessments.

Local treatment of surgery and/or radiotherapy follows VIDE or VDC/IE induction chemotherapy, and whenever feasible, surgery proceeds after cycle 6 of VIDE (Arm A) or cycle 9 VDC/IE (Arm B) on haematological recovery. Decisions on treatment of the primary tumour are individualised as is necessary in this disease. Consolidation chemotherapy is administered according to treatment arm randomised to and whether the patient has regional lymph node involvement or metastatic disease, or risk group localised disease (good risk or poor risk). The definition localised poor risk and good risk is based on presence or absence of a combination of factors, whether there is; resection at diagnosis, tumour volume $\geq 200 \mathrm{ml}$, pre-operative radiotherapy, histological response ( $\geq 10 \%$ viable tumour), unresectable tumour treated with radiotherapy alone, and volume $<200 \mathrm{ml}$ with poor radiological response.

Peripheral blood stem cell (PBSC) mobilisation and harvesting is recommended after VIDE/VDC/IE chemotherapy if defined as poor risk localised disease. PBSC mobilisation and harvesting should be performed according to institutional guidelines. BuMel treatment is contraindicated for patients where radiotherapy is required to the central axial sites (spine, sacrum, pelvis) or when lung or bowel are within the radiotherapy treatment fields (the protocol includes specific criteria regarding doses). Radiotherapy is recommended to be given concurrently with consolidation chemotherapy to the primary site. In patients with pulmonary and/or pleural metastatic disease whole lung radiotherapy is recommended to be given on completion of consolidation chemotherapy. Radiotherapy to bony metastases may be given either during consolidation or at the end. At the end of treatment patients who received radiotherapy only as local control and who had residual disease pre-radiotherapy, an end of treatment MRI or CT scan should be performed. If the end of treatment scan shows residual disease, another scan should be performed six months after the end of treatment. After treatment, patients will be followed up with clinical evaluation and scanning for a minimum of 5 years, or until disease progression or death if sooner. Patient data is collected on the eRDC using a series of case report forms (CRFS), and follow-up forms are requested annually following the completion of treatment in order to track patient's status.

Patients are also asked to optionally consent to additional biological studies. Participation involves donating blood samples at multiple time points throughout the trial, and agreeing to any remaining bone marrow and diagnostic tumour biopsy tissue taken as routine practise being used for research purposes.

\section{Statistical considerations}

The target is to recruit a minimum of 600 patients to R1. The R2 target is a minimum of 300 patients. An analysis of R2 will also be done in conjunction with the German Ewing 2008 trial which will have a similar or greater number of patients, giving a total of about 600 patients (it is estimated 300 will come from EE2012 and 300 from Ewing 2008). It is anticipated that it will take at least 5 years to reach the accrual targets. Patients will be followed up for progression and death until all trial objectives have been met. The first main analysis will be performed once all patients have a minimum of 2 years follow-up. For each randomisation, the main analyses will be intention-to-treat (ITT) with all patients analysed in the arm to which allocated at randomisation. 
Due to the rarity of ESFT, and a restricted ability to randomise sufficient numbers of patients for a conventional design (with 2-sided alpha $=0.05$, power $=80 \%$ ), a Bayesian approach has been taken to the analysis of R1 which makes no prior assumptions that one chemotherapy arm is likely to be better than the other. This design will be reported in detail elsewhere.

For R2 conventional statistical analyses will be performed: Kaplan-Meier life tables will be constructed for time-to-event data (with date of randomisation as reference time point) and arms will be compared by means of the logrank test; continuous variables will be compared across arms by means of t-tests or Wilcoxon tests as appropriate. Multivariable analysis using Cox regression will be used to adjust for baseline co-variates as appropriate. Heterogeneity of the treatment effect according to these factors will be evaluated. As well as by individual trial, analyses of the zoledronic acid randomisation will be performed on the total data set for both trials combined (with stratification by trial).

\section{Discussion}

Internationally, the standard drug treatment of ESFT is not defined. This study aims to address this and establish a "standard regimen" of chemotherapy, taking into account both clinical outcomes (EFS and OS) and toxicity. This new "standard regimen" will then form the backbone of future international studies in ESFT, enhancing opportunities for collaboration and thus hastening progress in improving outcomes from this rare disease. In addition this study will provide evidence to establish whether add-on treatment with a novel agent in this disease setting, zoledronic acid, is of benefit for ESFT patients. Due to the rarity of the tumour and the need for timely answers, the study has a pragmatic statistical design, accepting that it is not possible to reach conventional levels of reliability within a reasonable time frame, and will recruit patients across multiple European countries.

Although these tumours are the second commonest malignant bone tumour in children, adolescents and young adults, they remain rare tumours (less than 70 cases per year in the UK and 100 in France) and hence any randomised trial must be international. Setting up and activating large international trials is a complex process, involving multiple institutions each with their own local practises, and requires acquiring approvals from numerous regulatory bodies across the participating countries. However it is anticipated that the knowledge, experience and relationships formed through activating EE2012 internationally will be of benefit to any future trials established by the EEC and lead to shorter trial set-up times and therefore quicker answers to important therapeutic questions.

\section{Trial status}

The trial is opened and the first patient entered March 2014. At the time of manuscript submission (April 2019) the 639 and 242 patients had been recruited into R1 into R2 respectively and recruitment to R1 is anticipated to continue until 30th September 2019 (R2 will then approximately 5 months later to allow the final patient into R1 to enter R2). The current version of the protocol is version 5.0 dated 2nd June 2017. 


\section{List Of Abbreviations}

ITT intention-to-treat

EEC EURO EWING Consortium

ESFT Ewing sarcoma family of tumours

EFS event-free survival

OS overall survival

\section{Declarations}

\section{Ethics approval, consent to participate and confidentiality}

The trial was initially approved by NRES Committee North West - Greater Manchester Central on 1st February 2013. Since then there have been 11 approved substantial amendments and the latest version of the protocol (version 5.0) was approved by the ethics committee on 7th August 2017. This latest amendment to protocol version 5.0 was to change the consolidation treatment for patients with poor risk localised disease to VAI plus BuMel, following the results of the earlier trial EURO-E.W.I.N.G. 99 becoming available demonstrating this treatment to be superior. Please note that the dates of ethical approvals are for the submissions made by the Coordinating Sponsor in the United Kingdom, each NCC has to submit to and gain approvals from the relevant regulatory bodies in their country(ies). Central ethical approval for the whole UK was confirmed by the NRES Committee North West - Greater Manchester Central (ref approval no. 12/NW/0827) and we will not begin recruiting at other centres and in other countries until the relevant country specific and local ethical approval has been obtained.

Written informed consent will be obtained from each patient and/or parent/legal guardian for each randomisation prior to performing any trial related procedure. At trial entry, the patient and/or parent/legal guardian will also be given the option of consenting to the collection, storage and analysis of additional tumour, blood and bone marrow samples for use in biological studies associated with the trial, but this does not affect whether they can enter the trial. Patients have the right to withdraw from the study at any time and for any reason without prejudice to their future medical care.

With the patient's consent (and where national legislation/guidance permits) their full name, date of birth, hospital number, medical practitioner details and national registry numbers (e.g. National Health Service (NHS) Number in the UK) will be collected at trial entry to allow long-term follow-up via other health care professionals (e.g. patient's medical practitioner) and national cancer registries. Personal data recorded on all documents will be regarded as strictly confidential and will be handled and stored in accordance with the relevant data protection legislation in the member state. Patients will be identified using only their unique trial number and, if national legislation permits, their initials and date of birth on the header section of the eRDC screens and in correspondence between the applicable NCC and participating sites. 


\section{Consent for publication}

Not applicable.

\section{Availability of data and materials}

No data is presented in this manuscript. The materials described can be obtained by contacting the corresponding author.

\section{Competing interests}

The authors declare that they have no competing interests.

\section{Funding}

This project has received funding from the European Union's Seventh Framework Programme for research, technological development and demonstration under grant agreement $n^{\circ} 602856$. The NCC in France, CLB, receives additional funding from SFCE and Ligue contre le cancer. The Coordinating Sponsor, United Kingdom is funded by Cancer Research UK (grant award reference C5952/A14745). The funding bodies had no role in the design of the study and collection, analysis, and interpretation of data and in writing the manuscript.

\section{Authors' contributions}

All authors (J.A., V.M., P.M-B., N.G., V.L., J.M-B., A.S., H.G., C.O., S.K., M.F-P., N.F., A.E., S.S., J.W., K.W. and B.B.) approved the final manuscript.

\section{Acknowledgements}

We would like to acknowledge members of the EEC and all investigators and trial staff at participating trial centres. We also acknowledge colleagues at EEC partner organisations (UoB, CLB, GEIS, OLCH, EORTC) who support the trial within their institution.

\section{References}

1. Ambros, I.M., et al., MIC2 is a specific marker for Ewing's sarcoma and peripheral primitive neuroectodermal tumors. Evidence for a common histogenesis of Ewing's sarcoma and peripheral primitive neuroectodermal tumors from MIC2 expression and specific chromosome aberration. Cancer, 1991. 67(7): p. 1886-93.

2. Aurias, A., et al., Translocation involving chromosome 22 in Ewing's sarcoma. A cytogenetic study of four fresh tumors. Cancer Genet Cytogenet, 1984. 12(1): p. 21-5. 
3. Delattre, O., et al., The Ewing family of tumors--a subgroup of small-round-cell tumors defined by specific chimeric transcripts. N Engl J Med, 1994. 331(5): p. 294-9.

4. Dockhorn-Dworniczak, B., et al., Diagnostic value of the molecular genetic detection of the $t(11 ; 22)$ translocation in Ewing's tumours. Virchows Arch, 1994. 425(2): p. 107-12.

5. Kovar, H., et al., Overexpression of the pseudoautosomal gene MIC2 in Ewing's sarcoma and peripheral primitive neuroectodermal tumor. Oncogene, 1990. 5(7): p. 1067-70.

6. Turc-Carel, C., et al., [Chromosomal translocation $(11 ; 22)$ in cell lines of Ewing's sarcoma]. C R Seances Acad Sci III, 1983. 296(23): p. 1101-3.

7. Whang-Peng, J., et al., Chromosome translocation in peripheral neuroepithelioma. N Engl J Med, 1984. 311(9): p. 584-5.

8. Spunt, S.L., et al., Selective use of whole-lung irradiation for patients with Ewing sarcoma family tumors and pulmonary metastases at the time of diagnosis. J Pediatr Hematol Oncol, 2001. 23(2): p. 938.

9. Oberlin, O., et al., Impact of high-dose busulfan plus melphalan as consolidation in metastatic Ewing tumors: a study by the Societe Francaise des Cancers de l'Enfant. J Clin Oncol, 2006. 24(24): p. 39974002.

10. Womer, R.B., et al., Randomized controlled trial of interval-compressed chemotherapy for the treatment of localized Ewing sarcoma: a report from the Children's Oncology Group. J Clin Oncol, 2012. 30(33): p. 4148-54.

11. Powles, T., E. McCroskey, and A. Paterson, Oral bisphosphonates as adjuvant therapy for operable breast cancer. Clin Cancer Res, 2006. 12(20 Pt 2): p. 6301s-6304s.

12. Rosen, L.S., et al., Long-term efficacy and safety of zoledronic acid compared with pamidronate disodium in the treatment of skeletal complications in patients with advanced multiple myeloma or breast carcinoma: a randomized, double-blind, multicenter, comparative trial. Cancer, 2003. 98(8): p. 1735-44.

13. Berry, S., et al., The use of bisphosphonates in men with hormone-refractory prostate cancer: a systematic review of randomized trials. Can J Urol, 2006. 13(4): p. 3180-8.

14. Sonnemann, J., et al., The bisphosphonate pamidronate is a potent inhibitor of Ewing's sarcoma cell growth in vitro. Anticancer Drugs, 2003. 14(9): p. 767-71.

15. Zhou, Z., et al., Zoledronic acid inhibits primary bone tumor growth in Ewing sarcoma. Cancer, 2005. 104(8): p. 1713-20. 
16. Odri, G.A., et al., Zoledronic acid as a new adjuvant therapeutic strategy for Ewing's sarcoma patients. Cancer Res, 2010. 70(19): p. 7610-9.

\section{Tables}

\begin{tabular}{|c|c|}
\hline Randomisation 1 & Randomisation 2 \\
\hline \multicolumn{2}{|c|}{ Inclusion criteria } \\
\hline $\begin{array}{l}\text { - } \text { Any histologically and genetically } \\
\text { confirmed ESFT of bone or soft tissue, or } \\
\text { round cell sarcomas 'Ewing's-like' but } \\
\text { negative for EWSR } 1 \text { gene rearrangement. } \\
\text { - } \quad \text { Age }>2 \text { years and }<50 \text { years } \\
\text {. } \text { Randomisation } \leq 45 \text { days after diagnostic } \\
\text { biopsy/surgery } \\
\text {. } \text { Patient medically fit to receive trial } \\
\text { treatment }\end{array}$ & $\begin{array}{l}\text { Age }>5 \text { years } \\
\text { Localised tumour, or metastatic disease and/or } \\
\text { regional lymph node(s) involvement only at } \\
\text { diagnosis and at least partial response of } \\
\text { metastases and/or regional lymph node(s) } \\
\text {. Consolidation chemotherapy as per protocol } \\
\text { intended } \\
\text {. } \text { Medically fit to receive zoledronic acid }\end{array}$ \\
\hline \multicolumn{2}{|c|}{ Exclusion criteria } \\
\hline $\begin{array}{l}\text { - Other malignancy } \\
\text {. } \text { Pregnant or breastfeeding women }\end{array}$ & $\begin{array}{l}\text { History of dental surgery in the previous } 6 \text { months } \\
\text { or planned dental surgery during treatment or } \\
\text { within } 6 \text { months after the end of treatment. } \\
\text {. History of jaw fracture } \\
\text { - Ewing's tumour of the maxilla or of the mandible } \\
\text { - Progression of the primary tumour or appearance } \\
\text { of new lesions }\end{array}$ \\
\hline
\end{tabular}

Table 1: Inclusion and exclusion criteria

\section{Supplementary File Legend}

Supplementary table 1: Summary of treatment details the schedules of administration of cycles of chemotherapy and zoledronic acid.

\section{Figures}




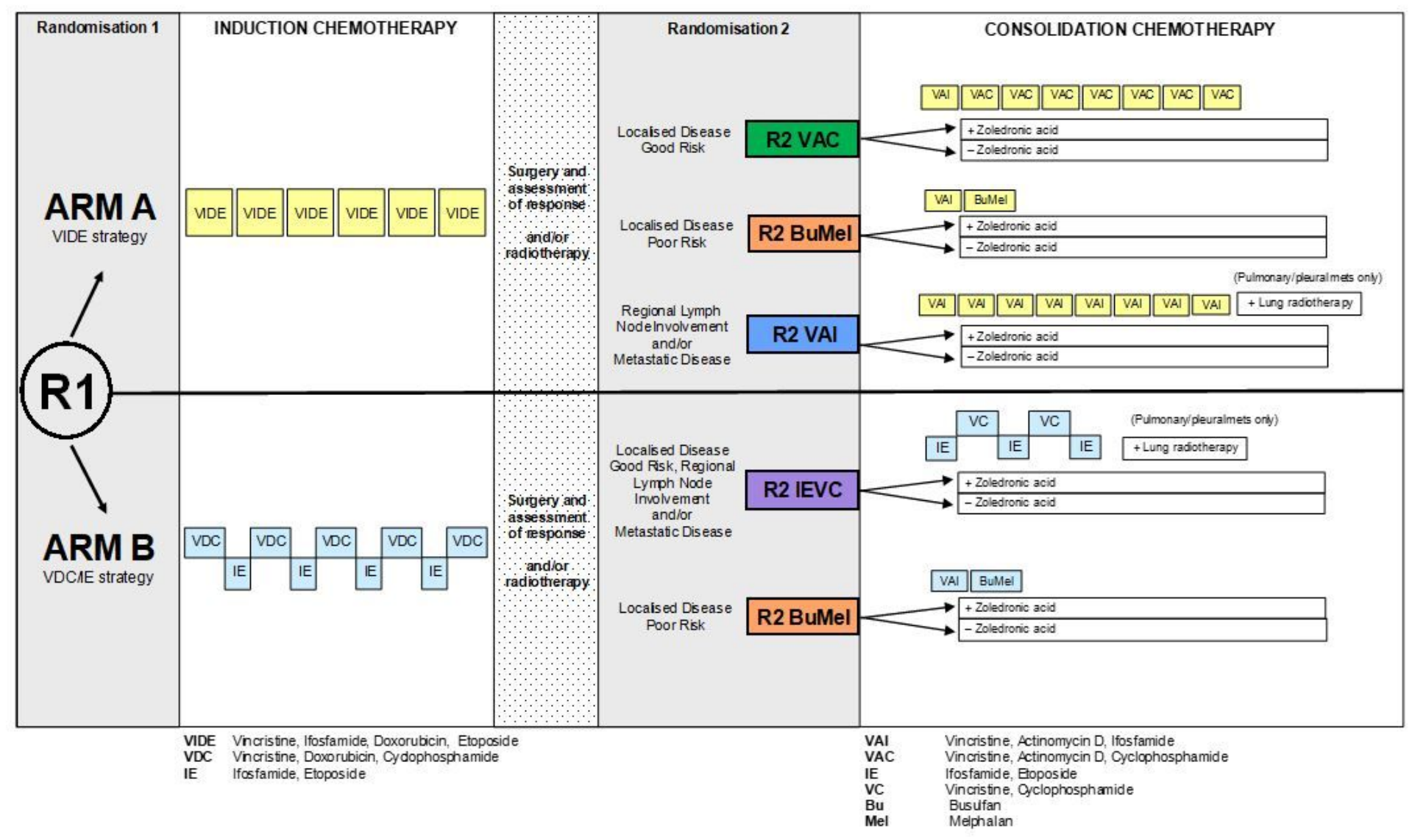

Figure 1

Trial Schema 


\begin{tabular}{|c|c|c|c|c|c|c|c|}
\hline & \multirow[t]{2}{*}{ Enrolment } & \multirow[t]{2}{*}{ Allocation } & \multicolumn{3}{|c|}{ Treatment } & \multirow{2}{*}{$\begin{array}{l}\text { End of treatment } \\
\qquad \text { (EOT) }\end{array}$} & \multirow{2}{*}{$\begin{array}{l}6 \text { months } \\
\text { after EOT }\end{array}$} \\
\hline & & & R1 Induction & $\begin{array}{c}\text { Post } \\
\text { induction }\end{array}$ & $\begin{array}{l}\text { R1 Consolidation } \\
\text { and/or R2 treatment }\end{array}$ & & \\
\hline TIMEPOINT & $-t_{1}$ & 0 & $t_{1}$ & $t_{2}$ & $t_{3}$ & $\mathbf{t}_{4}$ & $t_{5}$ \\
\hline \multicolumn{8}{|l|}{ ENROLMENT: } \\
\hline Eligibility screen & $\mathrm{X}$ & & & $X(R 2)$ & & & \\
\hline Informed consent & $x$ & & & $X(R 2)$ & & & \\
\hline Allocation & & $\mathrm{x}$ & & $\mathrm{X}(\mathrm{R} 2)$ & & & \\
\hline \multicolumn{8}{|l|}{ INTERVENTIONS: } \\
\hline R1: Arm A: VIDE followed by VAI/VAC & & & $x$ & & $\mathrm{X}$ & & \\
\hline R1: Arm B: VDC followed by IE/VC & & & $x$ & & $\mathrm{X}$ & & \\
\hline R2: Zoledronic acid or not & & & & & $X(R 2)$ & & \\
\hline \multicolumn{8}{|l|}{ ASSESSMENTS: } \\
\hline MRI or CT & $\mathrm{x}$ & & $\mathrm{x}$ & $\mathrm{x}$ & & $x^{b}$ & $\mathrm{X}^{\mathrm{c}}$ \\
\hline Chest CT scan & $x$ & & $x$ & $x^{a}$ & & & \\
\hline Radionuclide scan of skeleton (or PET scan) & $x$ & & & & & & \\
\hline $\begin{array}{l}\text { Estimation of primary tumour volume (and } \\
\text { lymph node involvement if applicable) }\end{array}$ & $x$ & & & & & & \\
\hline Assessment of adverse events & & & $\mathrm{x}$ & & $\mathrm{x}$ & & \\
\hline
\end{tabular}

${ }^{2}$ patients with pulmonary/pleural metastatic disease only

${ }^{b}$ patients who had radiotherapy only and who had residual disease pre-radiotherapy

' patients who had residual disease at end of treatment

\section{Figure 2}

Summary schedule of enrolment, interventions, and assessments.

\section{Supplementary Files}

This is a list of supplementary files associated with this preprint. Click to download.

- supplement1.docx

- supplement2.pdf 